\title{
Vulnerabilität und Resilienz im Verkehrssektor
}

\author{
Den Begriffen Resilienz und Vulnerabilität wird \\ in der verkehrswissenschaftlichen Forschung \\ bisher nur eine geringe Bedeutung beigemessen. \\ Auch in der Praxis lassen sich kaum konsistente \\ Resilienzstrategien finden. Doch die zunehmende \\ digitale Vernetzung erfordert neue Konzepte. \\ Von Marc Schelewsky und Weert Canzler
}

A lle Welt spricht von „autonomous driving“ oder „connected cars“. Das ist aber nur die verkürzte automobile Seite der informations- und kommunikationstechnischen Aufrüstung im Verkehr, die wir derzeit erleben. Sie ist eng mit der Illusion verbunden, im motorisierten Individualverkehr einfach so weitermachen zu können wie bisher. Nur eben künftig digital unterstützt. Die andere - und nicht selten mit Hoffnungen auf eine „Verkehrswende“ verbundene Seite - ist die durch Informations- und Kommunikationssysteme (IuK) gestützte neue Intermodalität. Also die bedienungsfreundliche Verknüpfung der verschiedenen Verkehrsmittel, „Mobilitäts-App“ und „Digitaler Mobilitäts-Assistent“ heißen die Zauberworte (Canzler/Knie 2016). Auf der Jahrestagung des Verbandes deutscher Verkehrsunternehmen (VDV) 2017 in Hannover wurde die App „Mobility Inside“ vorgestellt, die als digitale Plattform die Angebote des Öffentlichen Verkehrs umfassend integrieren und diese mit weiteren Mobilitätsangebote wie Car-, Bike- oder Ridesharing verknüpfen soll. Das Ganze soll dabei für die Kundschaft so einfach wie möglich sein: Einmal registrieren - alles nutzen.

\section{Gefährliche Verkehrswende}

Auf technischer und unternehmerischer Seite erfordern solche Vernetzungen umfassende Eingriffe in die bestehenden IT-Systeme der Verkehrsunternehmen. Es müssen neue Schnittstellen geschaffen und analoge Prozesse digitalisiert werden. Nicht zuletzt stellt der Austausch von Daten oder die Nutzung von Diensten in fremden Systemen hohe Anforderungen an die Sicherheit der jeweiligen Systeme. Das gilt umso mehr, als auch Daten der Kund/innen inklusive Information zu den Bezahlvarianten (Kreditkarteninformationen) in dem nun vernetzten System hinterlegt werden. Durch die Vernetzung erhöht sich die Zahl der externen Zugriffs- und damit auch Angriffspunkte zwangsläufig. Das gilt gleichermaßen für den Betrieb (Ausfallsicherheit) als auch für die Nutzerdaten (Datenschutz und -sicherheit).
Aber wie „resilient“ sind nun diese umwelt- und verkehrspolitisch gewollten Vernetzungen? Resilienz wird dabei als ein „umfassender, holistischer Problemlösungsansatz verstanden (...), dessen Ziel es ist, die generelle Widerstandskraft und Regenerations- und Entwicklungsfähigkeit von natürlichen und gesellschaftlichen Systemen zu erhalten (Fekkak et al. 2016, S. 10 f.).“ Mit Blick auf den Verkehrssektor geht es um die Robustheit und Redundanz von Infrastrukturen, Fahrzeugen und IT-Systemen. Bisher ist der Begriff „Resilienz “ in der verkehrswissenschaftlichen Forschung nur wenig in Erscheinung getreten, was im Übrigen auch für andere sozio-technische Felder gilt (Maurer 2016). Eines der wenigen Beispiele ist das von der Bundesanstalt für Straßenwesen initiierte Forschungsprogramm AdSVIS (Adaption of the Road Infrastructure to Climate Change), das bereits zwischen 2009 und 2014 bearbeitet wurde und dabei in praxisorientierten Projekten wie RIVA (Risikoanalyse wichtiger Verkehrsachsen des Bundesfernstraßennetzes im Kontext des Klimawandels) ein Bewertungsschema für Risikopotenziale der Straßenverkehrsinfrastruktur entwickelte. Vergleichbare Projekte für den öffentlichen Verkehr sind dagegen nicht bekannt.

Die Gründe dafür sind vielfältig: Im Alltagsbetrieb der Unternehmen entstehen Maßnahmen zur Verringerung von Vulnerabilität und Erhöhung der Resilienz eher als Nebeneffekt denn als Folge strategischer Überlegungen und Planungen. Oftmals ist ein rein pragmatischer Ansatz handlungsleitend, wenn beispielsweise eine Trennung der verschiedenen IT-Systeme mehr Komfort bietet, da Anpassungen des Entertainment-Bereichs ohne Zustimmung der Genehmigungsbehörde vorgenommen werden können. Oft besteht zwar durchaus ein Bewusstsein für die Gefahren und Anforderungen, die die zunehmende Vernetzung der Einzelsysteme mit sich bringen, aber es fehlt das entsprechende Knowhow, diese Projekte mit der erforderlichen Expertise zu bearbeiten. Die dazu erforderlichen Kompetenzen und profunden Kenntnisse über verteilte IT-Architekturen, Schnittstellenstandards oder neue Datenbanksysteme sind oftmals nicht in ausreichendem Umfang vorhanden und müssten extern eingekauft werden. Zusammen mit den oftmals zusätzlich erforderlichen Investitionskosten in die IT-Infrastruktur, zum Beispiel für den Aufbau redundanter Systeme, überfordert das vor allem kleinere Unternehmen. Entsprechende Projekte werden dann gar nicht angegangen und man verbleibt in den bestehenden Strukturen. Betriebsrelevante IT-Systeme bleiben "gekapselt“ und werden bewusst nicht als Webservice für eine Vernetzung umgesetzt, um dadurch mehr Sicherheit zu gewährleisten. Dass diese Strategie 
der Unterlassung die Zukunftsfähigkeit des Unternehmens aufs Spiel setzt, scheint dabei nicht reflektiert zu werden beziehungsweise es werden keine Alternativen gesehen.

\section{Ansätze für ein starkes Verkehrssystem}

Was also ist zu tun? Generell gilt, „dass ein Verkehrssystem umso verletzbarer ist, je größer das Ausmaß an digitaler Technologie ist, das zu seiner Betriebsführung eingesetzt wird“ (Rammler 2014, S. 85). Die Themen Vulnerabilität und Resilienz sollten deshalb eine stärkere Verankerung innerhalb der verkehrswissenschaftlichen Forschung finden, mit dem Ziel, den Herausforderungen durch die Digitalisierung im Verkehrssektor angemessen zu begegnen und die Begriffe selber für die praktische Nutzung zu schärfen. Dabei sollte der Bezug zu übergeordneten oder angrenzenden Systemen erhalten bleiben (zum Beispiel Stadt- oder Energiesysteme). Zudem sind insbesondere die Schnittstellen an den Systemübergängen zu berücksichtigen und Konzepte gegen Kaskadeneffekte und Kettenreaktionen zu entwickeln, die dabei aber einer zunehmenden Vernetzung nicht hinderlich sind. Eine bloße Bearbeitung innerhalb der Disziplingrenzen birgt die Gefahr, dass Resilienz als systemübergreifende Herausforderung aus dem Blick gerät und vielmehr in getrennte, intradisziplinäre Diskurse und Einzelprobleme zerfällt. Abhängigkeiten zwischen Systemen werden dann zum blinden Fleck, wodurch die Vulnerabilität insgesamt erhöht wird (Pescaroli/Alexander 2016).

In Bezug auf das System „Stadt“ konstatiert dazu Beckmann (2013), dass „(...) die Vernetzung, die Wechselwirkungen und die positiven wie negativen Rückkopplungen systemanalytisch betrachtet werden (müssen). Es gilt ,systemkritische‘ aktive Einflussparameter zu ermitteln, welche die Systemstabilität gefährden können (Beckmann 2013, S. 12).“ Dies kann über Szenarien erfolgen, die einflussstarke Entwicklungen (Megatrends) abbilden und deren Wirkung auf das abhängige System modellieren und zudem die Grundlage bilden, um geeignete Gegenmaßnahmen zu entwickeln (ebd.). Leitend sollten dabei die Prinzipien Autarkie, Redundanz, Auswirkungsminimierung und Wiederherstellung der Systemkapazität sein (Randelhoff 2013). In der Praxis ergeben sich dann Standards, Checklisten oder Leitfäden, die im Hinblick auf konkrete Problemstellungen entwickelt werden. Hinzu kommt die offene Frage, ob eine zunehmende Transparenz von Nutzerinnen und Nutzern intermodaler Services und eine auf persönlichen Profilen beruhende individualisierte Mobility as a Service weitgehend akzeptiert werden oder ob es - möglicherweise in der Folge von spektakulären Missbrauchsfällen - zu Akzeptanzproblemen kommen kann, die das Vernetzungsmodell insgesamt gefährden.

Das Nichtwissen und die Unsicherheit sind weitverbreitet, andererseits sind die ökologischen Vorteile effizienter intermodaler Verkehrsangebote, die digital basiert sind, unübersehbar und können nicht länger ignoriert werden. Für den Praxisbezug und die Berücksichtigung interdisziplinärer Perspektiven stellen Reallabore einen geeigneten Ansatz dar, da sich hier unterschiedliche Handlungsebenen, Akteure/innen und Disziplinen durch die Integrität des Raumes bündeln lassen (De Flander et al. 2014). Die entwickelten Konzepte, Verfahren, Checklisten et cetera, aber auch sicherheitsrelevante Strukturen und IT-Systeme sollten systematisch innerhalb dieser Reallabore entwickelt, getestet und schließlich auf Praxistauglichkeit überprüft und gegebenenfalls angepasst werden. Resilienz in einer künftigen digitalen und im Übrigen auch postfossilen Mobilität ist nicht nur als Aufgabe der strategischen Planung von Unternehmen zu betrachten, sondern eine gesamtgesellschaftliche Anforderung, die sich gleichermaßen an Wissenschaft, Politik, Verwaltung, Unternehmen und auch Zivilgesellschaft richtet. Auch dafür besteht in Reallaboren ein geeigneter Ausgangspunkt.

\section{Literatur}

Beckmann, K. J. (2013): Resilienz - Eine neue Anforderung im Zusammenhang mit nachhaltiger Stadtentwicklung? In: Beckmann, K. J. (Hrsg.): Jetzt auch noch resilient? Anforderungen an die Krisenfestigkeit der Städte. difu-Impulse 04/2013. Berlin. 7-13.

Canzler, W./Knie, A. (2016): Die digitale Mobilitätsrevolution. Vom Ende des Verkehrs, wie wir ihn kannten. München, oekom verlag.

De Flander, K. et al. (2014): Resilienz und Reallabore als Schlüsselkonzepte urbaner Transformationsforschung. Zwölf Thesen. In: GAIA - Ecological Perspectives for Science and Society 23/3: 284-286.

Fekkak, M. et al. (2016): Resiliente Stadt - Zukunftsstadt. Forschungsgutachten des Wuppertal Institut im Auftrag des Ministeriums für Bauen, Wohnen, Stadtentwicklung und Verkehr des Landes Nordrhein-Westfalen (MBWSV). O. O.

Maurer, A. (Hrsg.) (2016): New Perspectives on Resilience in Socio-Economic Spheres. Wiesbaden, SpringerVS.

Pescaroli, G./Alexander, D. (2016): Critical infrastructure, panarchies and the vulnerability paths of cascading disasters. In: Natural Hazards 82/1: 175-192.

Rammler, S. (2014): Schubumkehr. Zukunft der Mobilität. Frankfurt a. M., Fischer Taschenbuch.

Randelhoff, M. (2013): Resiliente Infrastrukturen und Städte: Kritikalität und Interdependenzen. www.zukunft-mobilitaet.net/40882/analyse/ resilienz-infrastruktur-stadt-wirtschaft-zukunft-resiliente-infrastrukturen/

\section{AUTOREN + KONTAKT}

Marc Schelewsky ist Team-Koordinator für digitale Lösungen am Innovationszentrum für Mobilität und gesellschaftlichen Wandel (InnoZ).

Innovationszentrum für Mobilität und gesellschaftlichen Wandel (InnoZ), EUREF-Campus 16, 10829 Berlin. E-Mail: marc.schelewsky@innoz.de, Website: www.innoz.de

Dr. Weert Canzler ist Sprecher des LeibnizForschungsverbundes Energiewende am Wissenschaftszentrum Berlin für Sozialforschung (WZB).

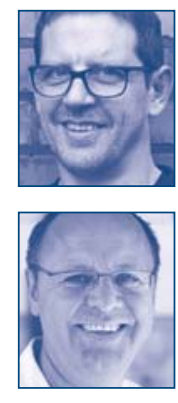

Wissenschaftszentrum Berlin für Sozialforschung (WZB), Reichpietschufer 50, 10785 Berlin.

E-Mail:weert.canzler@wzb.eu, Website: www.wzb.eu 